\title{
A Simple Proof and Refinement of Wielandt's Eigenvalue Inequality
}

\author{
Lutz Dümbgen
}

published 1995 in Statist. Prob. Letters 25, 113-115

\begin{abstract}
Wielandt (1967) proved an eigenvalue inequality for partitioned symmetric matrices, which turned out to be very useful in statistical applications. A simple proof yielding sharp bounds is given.
\end{abstract}

Keywords and phrases: eigenvalue inequality, partitioned matrix

Correspondence to: Lutz Duembgen, Institute of Mathematical Statistics and Actuarial Science, University of Bern, Sidlerstrasse 5, CH-3012 Bern, Switzerland; e-mail: duembgen@stat.unibe.ch 
Let $A \in \mathbf{R}^{p \times p}$ be a symmetric matrix of the form

$$
A=\left(\begin{array}{cc}
B & C \\
C^{\prime} & D
\end{array}\right)
$$

with $B \in \mathbf{R}^{r \times r}, C \in \mathbf{R}^{r \times s}$ and $D \in \mathbf{R}^{s \times s}$ such that

$$
\lambda_{r}(B)>\lambda_{1}(D)
$$

generally $\lambda_{1}(E) \geq \lambda_{2}(E) \geq \cdots \geq \lambda_{q}(E)$ denote the ordered eigenvalues of a symmetric matrix $E \in \mathbf{R}^{q \times q}$. Wielandt (1967) showed that the eigenvalues of $A$ can be approximated by the eigenvalues of $B$ and $D$ in the following sense:

$$
\begin{aligned}
0 \leq \lambda_{i}(A)-\lambda_{i}(B) & \leq \frac{\lambda_{1}\left(C C^{\prime}\right)}{\lambda_{i}(B)-\lambda_{1}(D)} \text { for } 1 \leq i \leq r \text { and } \\
0 \leq \lambda_{j}(D)-\lambda_{r+j}(A) & \leq \frac{\lambda_{1}\left(C C^{\prime}\right)}{\lambda_{r}(B)-\lambda_{j}(D)} \text { for } 1 \leq j \leq s .
\end{aligned}
$$

These inequalities can be used to compute derivatives and pseudo-derivatives of eigenvalues. They are also very useful in statistical problems involving eigenvalues of random symmetric matrices; see Eaton and Tyler (1991, 1994). In my opinion the original proof, described in Eaton and Tyler (1991), is somewhat complicated. The main ingredient seems to be the Courant-Fischer minimax representation

$$
\lambda_{k}(E)=\max _{\mathbf{V}: \operatorname{dim}(\mathbf{V})=k} \min _{v \in \mathbf{V}: v^{\prime} v=1} v^{\prime} E v \text { for } 1 \leq k \leq q,
$$

where $\mathbf{V}$ stands for a linear subspace of $\mathbf{R}^{q}$; see section 1f.2 of Rao (1973). In this note (2) is used directly to derive the following refinement of (1):

Theorem. For $1 \leq i \leq r$,

$$
0 \leq \lambda_{i}(A)-\lambda_{i}(B) \leq \sqrt{\frac{\left(\lambda_{i}(B)-\lambda_{1}(D)\right)^{2}}{4}+\lambda_{1}\left(C C^{\prime}\right)}-\frac{\lambda_{i}(B)-\lambda_{1}(D)}{2},
$$

and for $1 \leq j \leq s$,

$$
0 \leq \lambda_{j}(D)-\lambda_{r+j}(A) \leq \sqrt{\frac{\left(\lambda_{r}(B)-\lambda_{j}(D)\right)^{2}}{4}+\lambda_{1}\left(C C^{\prime}\right)}-\frac{\lambda_{r}(B)-\lambda_{j}(D)}{2} .
$$




\section{Remark 1: Since}

$$
\sqrt{\alpha^{2} / 4+\beta^{2}}-\alpha / 2 \leq \min \left\{\beta, \beta^{2} / \alpha\right\} \forall \alpha, \beta>0
$$

this result implies Wielandt's bounds (1).

Remark 2: The upper bounds are sharp. For if $p=2$ one can compute the eigenvalues of $A$ explicitly and obtains

$$
\lambda_{1}(A)-\lambda_{1}(B)=\lambda_{1}(D)-\lambda_{2}(A)=\sqrt{\frac{(B-D)^{2}}{4}+C^{2}}-\frac{B-D}{2} .
$$

For general $p$ one has to consider diagonal matrices $B, D$ and suitable matrices $C$ with only one nonzero coefficient.

Proof of the Theorem: One easily verifies that the asserted inequalities are invariant under the transformation $A \mapsto A-\lambda_{1}(D) I$, where $I$ is the identity matrix in $\mathbf{R}^{p \times p}$. Therefore one may assume without loss of generality that $\lambda_{1}(D)=0$.

For $1 \leq i \leq r$ it follows from (2) that

$$
\lambda_{i}(A) \geq \max _{\mathbf{V} \subset \mathbf{R}^{r} \times\{0\}: \operatorname{dim}(\mathbf{V})=i} \min _{v \in \mathbf{V}: v^{\prime} v=1} v^{\prime} A v=\lambda_{i}(B) .
$$

On the other hand, let $\mathbf{W}$ be an $i$-dimensional subspace of $\mathbf{R}^{p}$ such that

$$
\lambda_{i}(A)=\min _{v \in \mathbf{W}: v^{\prime} v=1} v^{\prime} A v .
$$

If $v \in \mathbf{R}^{p}$ is written as $v=\left(v_{(1)}^{\prime}, v_{(2)}^{\prime}\right)^{\prime}$ with $v_{(1)} \in \mathbf{R}^{r}$ and $v_{(2)} \in \mathbf{R}^{s}$, then

$$
\mathbf{W}_{(1)}=\left\{v_{(1)}: v \in \mathbf{W}\right\}
$$

is an $i$-dimensional subspace of $\mathbf{R}^{r}$. For if $\operatorname{dim}\left(\mathbf{W}_{(1)}\right)<i$, then $w_{(1)}=0$ for some unit vector $w \in \mathbf{W}$, and

$$
\lambda_{i}(A) \leq w^{\prime} A w=w_{(2)}^{\prime} D w_{(2)} \leq 0,
$$

which would contradict (3). Any unit vector $v \in \mathbf{W}$ can be written as

$$
v=\sqrt{(1+\rho) / 2} u_{(1)}+\sqrt{(1-\rho) / 2} u_{(2)}
$$


for unit vectors $u_{(1)} \in \mathbf{W}_{(1)}, u_{(2)} \in \mathbf{R}^{s}$ and some $\rho \in[-1,1]$. Then

$$
\begin{aligned}
v^{\prime} A v & =(1+\rho) u_{(1)}^{\prime} B u_{(1)} / 2+\sqrt{1-\rho^{2}} u_{(1)}^{\prime} C u_{(2)}+(1-\rho) u_{(2)}^{\prime} D u_{(2)} / 2 \\
& \leq(1+\rho) u_{(1)}^{\prime} B u_{(1)} / 2+\sqrt{1-\rho^{2}} \sqrt{\lambda_{1}\left(C C^{\prime}\right)} \\
& =u_{(1)}^{\prime} B u_{(1)} / 2+\left(\rho, \sqrt{1-\rho^{2}}\right)\left(\begin{array}{c}
u_{(1)}^{\prime} B u_{(1)} / 2 \\
\sqrt{\lambda_{1}\left(C C^{\prime}\right)}
\end{array}\right) \\
& \leq u_{(1)}^{\prime} B u_{(1)} / 2+\sqrt{\left(u_{(1)}^{\prime} B u_{(1)}\right)^{2} / 4+\lambda_{1}\left(C C^{\prime}\right)} .
\end{aligned}
$$

Consequently, since $H(x):=x / 2+\sqrt{x^{2} / 4+\lambda_{1}\left(C C^{\prime}\right)}$ is nondecreasing in $x \geq 0$,

$$
\begin{aligned}
\lambda_{i}(A) & \leq \min _{u_{(1)} \in \mathbf{W}_{(1)}: u_{(1)}^{\prime} u_{(1)}=1} H\left(u_{(1)}^{\prime} B u_{(1)}\right) \\
& =H\left(\min _{u_{(1)} \in \mathbf{W}_{(1)}: u_{(1)}^{\prime} u_{(1)}=1} u_{(1)}^{\prime} B u_{(1)}\right) \\
& \leq H\left(\lambda_{i}(B)\right) \\
& =\lambda_{i}(B)+\sqrt{\left(\lambda_{i}(B)-\lambda_{1}(D)\right)^{2} / 4+\lambda_{1}\left(C C^{\prime}\right)}-\left(\lambda_{i}(B)-\lambda_{1}(D)\right) / 2 .
\end{aligned}
$$

Thus the first part of the theorem is true, and the second half follows by replacing $A$ with $-A$

\section{References}

Eaton, M.L. and D.E. Tyler (1991): On Wielandt's inequality and its application to the asymptotic distribution of the eigenvalues of a random symmetric matrix. Ann. Statist. 19, 260-271

Eaton, M.L. and D.E. Tyler (1994): The asymptotic distribution of singular values with applications to canonical correlations and correspondence analysis. $J$. Multivariate Anal. 50, 238-264

Rao, C.R. (1973): Linear Statistical Inference and Its Applications (2nd edition). Wiley, New York

Wielandt, H. (1967): Topics in the Analytic Theory of Matrices. (Lecture notes prepared by R. R. Meyer) Univ. Wisconsin Press, Madison 